\title{
Caracterización de la entonación venezolana a partir de un corpus obtenido con Map task
}

\author{
Josefa Dorta ${ }^{1}$ \\ Chaxiraxi Díaz \\ Universidad de La Laguna \\ Departamento de Filología Española \\ SEGAI Laboratorio de Fonética, España
}

\begin{abstract}
Resumen
En el proyecto de investigación "Estudio comparativo de la entonación $y$ del acento en zonas fronterizas del español" (FFI2014-52716-P), vinculado al proyecto internacional AMPER (Atlas Multimédia Prosodique de l'Espace Roman), se realiza una descripción prosódica del español venezolano ${ }^{2}$. En este trabajo abordamos un estudio entonativo fonético-fonológico del denominado corpus Map task de los puntos de encuesta de Mérida (Región de Los Andes), Barinas (Región de Los Llanos), Aragua (Región Central) y Bolívar
\end{abstract}

1 Para correspondencia, dirigirse a: Josefa Dorta (jdorta@ull.edu.es), ORCID 00000002-5204-8968, o a Chaxiraxi Díaz (chadiaz@ull.edu.es), ORCID 0000-0002-2854-5905. Universidad de La Laguna, Facultad de Humanidades (Sección Filología), Dpto. de Filología Española, Apartado 456, CP. 38200 La Laguna. S/C de Tenerife, Canarias, España.

2 El proyecto de referencia está centrado en la descripción y comparación de cinco variedades del español que son, además de la venezolana, la canaria, la cubana, la colombiana y la texana. En lo que respecta al venezolano, agradecemos la colaboración de Elsa Mora (Universidad de Los Andes). 
(Región Sur-Oriental), con el objetivo de comprobar si este tipo de corpus espontáneo valida las características prosódicas del corpus experimental descrito en trabajos anteriores. El análisis se realizó con subrutinas de MatLab y posteriormente fue supervisado con el programa Praat; los valores absolutos de F0, extraídos en el núcleo de la sílaba, fueron relativizados en semitonos, determinando su importancia perceptiva a partir del umbral diferencial de 1,5 semitonos.

Palabras clave: F0, entonación, corpus semiespontáneo, español venezolano.

\title{
Characterization OF VENEZUELAN INTONATION STEMMING FROM A CORPUS OBTAINED WITH MAP TASK
}

\begin{abstract}
In the investigation project "Comparative study of intonation and accent in Spanish frontier zones" (FFI2014-52716-P), vinculated to the international project AMPER (Atlas Multimédia Prosodique de l'Espace Roman), a prosodic description of Venezuelan Spanish is made. In this work, we make a phonetic-phonological intonative study of the corpus denominated Map task of Merida's survey points (Region of Los Andes), Barinas (Region of Los Llanos), Aragua (Central Region) and Bolivar (South-Oriental Region) with the objective of checking whether this type of sponataneous corpus validates the prosodic characteristics of the experimental corpus described in previous works. The analysis was realized with Matlab subroutines and was, later, survised with the Praat software; the absolute values of F0, extracted in the syllable nucleus, were relativized in semitones, determinind their perceptive importance from a differential threshold of 1,5 semitones.
\end{abstract}

Keywords: F0, intonation, semi-spontaneous corpus, Venezuelan Spanish.

Recibido: $26 / 03 / 20$

Aceptado: 05/04/21 


\section{INTRODUCCIÓN 3}

La geolingüística, surgida en el entorno de la dialectología a finales del siglo XIX, se ocupa de estudiar desde diversos puntos de vista (fonético, léxico, morfosintáctico, etc.) la variación lingüística en el espacio para reflejarla en los llamados atlas lingüísticos. En este marco, AMPER (Atlas Multimedia de Prosodia del Espacio Románico), nacido en el Centre de Dialectologie de l'Universitè Stendhal-Grenoble III $^{4}$, fue el primer atlas diseñado para describir la prosodia de las lenguas románicas y de sus variedades con el objetivo de plasmar los resultados en un soporte informático en forma de atlas multimedia.

De los dominios estudiados en AMPER (galorrománico, italorrománico, portugués, rumano, español, catalán, asturiano y gallego), las variedades del español se describen en España y en América: entre estas últimas está el español venezolano. Desde el punto de vista prosódico, hasta la llegada de AMPER, los estudios en Venezuela, circunscritos al ámbito dialectológico, no eran abundantes (Obregón 1981, Sosa 1991 y 1999, Chela Flores 1994, Chela Flores y Sosa 1999, Mora 1993, 1996 y 1997, Villamizar 1998) y no fue hasta hace pocos años cuando surgió el primer estudio de la entonación vinculado a AMPER (Mora, Méndez, Rodríguez y Rojas 2007) en el que se presenta un informe de avance del proyecto y los primeros resultados referidos a una mujer de una zona rural de Mérida. AMPER supone así un auge para los estudios de entonación en esta zona de América del Sur y, de hecho, se han realizado ya trabajos parciales en diferentes puntos como Mérida, Bolívar, Monagas, Caracas, Aragua, Barinas o Zulia (v. gr. Méndez, Mora y Rojas 2008, Méndez 2010, Dorta et al. 2013 b, Díaz, Muñetón Ayala y Dorta 2017, Dorta y Díaz 2018 b y c). Todos estos estudios tienen en común que han analizado voz femenina y un mismo tipo de corpus, el experimental,

Este trabajo se ha realizado en el marco del Proyecto de I+D Estudio comparativo de la entonación y del acento en zonas fronterizas del español (FFI2014-52716-P) del Programa estatal de fomento de la investigación científica y técnica de excelencia, subprograma estatal de generación del conocimiento [2015-2017, convocatoria 2014]. El presente trabajo está subvencionado también por el Excmo. Cabildo Insular de Tenerife, Innovación, Educación, Cultura y Deportes (Dirección de Cultura, Educación y Unidades Artísticas, resolución de 12 de diciembre de 2017).

4 En sus inicios AMPER fue coordinado internacionalmente por Michel Contini y Antonio Romano. Este último es el coordinador en la actualidad. Puede consultarse más información sobre el proyecto en el siguiente enlace: http://w3.u-grenoble3.fr/dialecto/ AMPER/BDAMPER/index.php. 
que es el que representa a AMPER en el atlas internacional que se difundirá por internet. A partir de tales estudios se ha podido establecer la siguiente diferenciación según el patrón final:

1) Regiones central (Caracas y Aragua) y Sur-oriental (Bolívar y Monagas): patrón descendente y circunflejo, en declarativas e interrogativas, respectivamente.

2) Región de Los Andes (Mérida): entonación circunfleja en las dos modalidades oracionales; se diferencian los patrones en el escalonamiento descendente de los picos de las declarativas.

3) Regiones de Zulia (Zulia) y Los Llanos (Barinas): entonación descendente en ambas modalidades. Estas se diferencian según Dorta y Díaz (2018 c) por "la distancia tonal que mantiene las interrogativas respecto de las declarativas".

En Díaz y Dorta (2018 b) se incluye por primera vez un estudio de la voz masculina venezolana (Mérida y Bolívar) y se revisan los puntos de encuesta estudiados con anterioridad para la femenina. Lo más destacado es que, en relación con el acento nuclear, las declarativas se caracterizan en los hombres por una entonación circunfleja como en la mujer de Mérida. Sin embargo, se concluye que el pico final raramente tiene relevancia perceptiva.

El estudio de los corpus más espontáneos considerados en AMPER (situacional, Map task y conversación libre) se ha iniciado recientemente (Aragua: Díaz y Dorta 2018 a; Bolívar: Díaz y Jorge 2019). En el presente trabajo se realiza un estudio entonativo fonético-fonológico del denominado corpus Map task en el que se han ampliado los puntos de encuesta estudiados hasta ahora para ver si se validan las características del corpus formal que hemos descrito en trabajos anteriores.

\section{MARCO METODOLÓGICO}

En relación con los puntos de encuesta, Mora (1996 y 1997) propone una división dialectal de Venezuela basada en características prosódicas:

1. Región Central: Distrito Capital y los Estados de Miranda, Vargas, Carabobo, Aragua, Lara, Yaracuy y Falcón.

2. Región de Los Llanos: Estados Portuguesa, Guárico, Cojedes, Apure y Barinas. 
3. Región Zulia: Estado Zulia.

4. Región Los Andes: Estados Táchira, Mérida y Trujillo.

5. Región Sur-Oriental: Estados Sucre, Nueva Esparta, Monagas, Anzoátegui, Delta Amacuro, Bolívar y Amazonas.

De acuerdo con esta propuesta, AMPER Venezuela se propuso comenzar a describir los siguientes puntos de encuesta en cada región: Mérida (Región de Los Andes), Barinas (Región de Los Llanos), Caracas y Aragua (Región Central), Bolívar y Monagas (Región Sur-Oriental) y Zulia (Región de Zulia). En este trabajo hemos elegido cuatro puntos: Aragua, Barinas, Mérida y Bolívar. En la figura 1 puede apreciarse su localización.

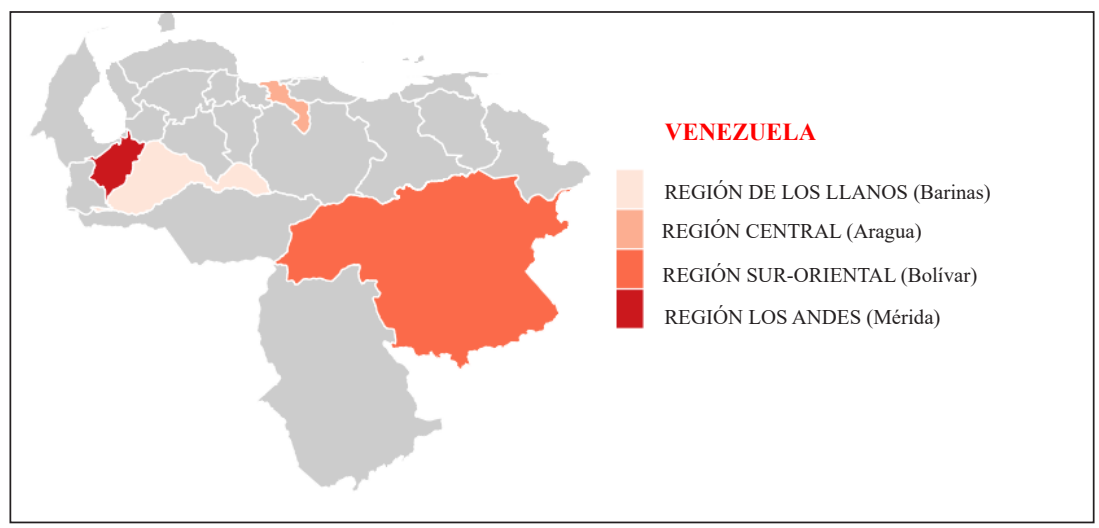

Figura 1. Mapa de Venezuela y puntos de encuesta estudiados

Con el propósito de comparar los patrones entonativos con los obtenidos previamente en habla formal, hemos elegido un total de cinco informantes en los que encontramos los mismos patrones ya descritos en trabajos anteriores (v. apartado 1), tres mujeres de Bolívar, Barinas y Aragua y dos hombres de Mérida y Bolívar, de modo que solo en este último punto hemos contado con los dos sexos debido a que hemos registrado distinto comportamiento tonal en el hombre y en la mujer:

- Para el patrón descendente en las declarativas y circunflejo en las interrogativas: las mujeres de Aragua y Bolívar.

- Para el patrón circunflejo en las dos modalidades: los hombres de Mérida y Bolívar.

- Para el patrón descendente en las dos modalidades: la mujer de Barinas. 
Todos los informantes son representativos de los cuatro puntos de encuesta señalados y, siguiendo la metodología de AMPER, sus condiciones sociolingüísticas son las siguientes: individuos sin estudios superiores de las zonas urbanas, de entre 25 y 55 años, nacidos y residentes en el punto de encuesta.

Véase en la figura $2^{5}$ un ejemplo de la configuración final de las curvas declarativas e interrogativas de estos informantes cuando el acento final es llano.

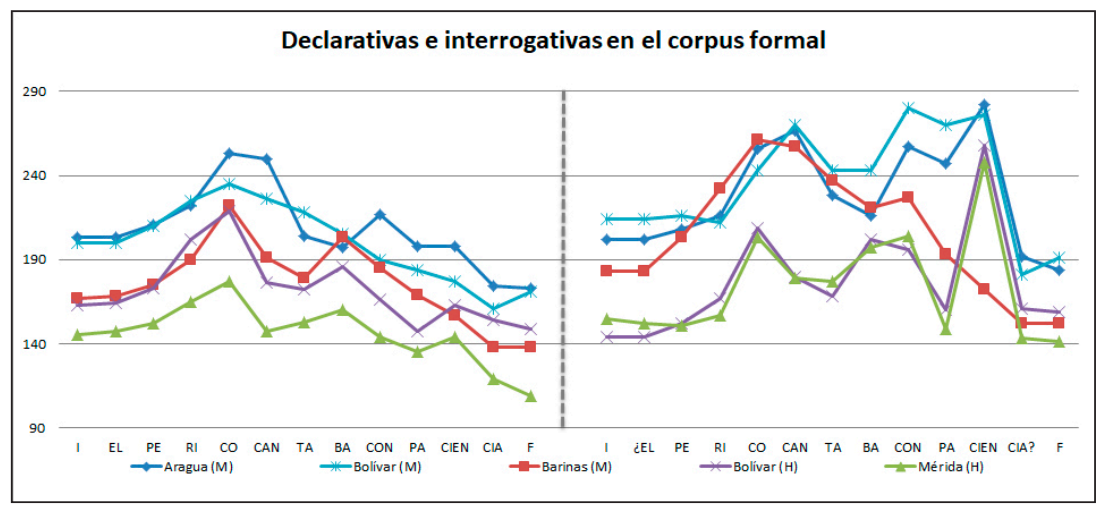

Figura 2. Patrones entonativos declarativos (izquierda) e interrogativos (derecha) en el corpus formal

En cuanto al corpus, hemos analizado uno de tipo Map task basado en el HCRC Map task Corpus, desarrollado por el Human Communication Research Center en 1991 (Anderson et al. 1991; The HCRC Map task Corpus 2008). Se obtiene mediante un sistema de recogida de datos a partir de dos mapas: el del informante -Instruction Follower-y el del entrevistador-Instruction Giver-; ambos deben salir de un punto geográfico y llegar a un destino determinado. Puesto que los dos mapas son similares, pero no idénticos, ello motivará una serie de preguntas y respuestas por parte de ambos intervinientes ${ }^{6}$. Puede verse un ejemplo de este tipo de mapas desarrollado por el Human Communication Research Center en la figura 3.

5 En la figura 2, M: mujer, H: hombre.

6 The Map Task is a cooperative task involving two participants. The two speakers sit opposite one another and each has a map which the other cannot see. One speaker-designated the Instruction Giver- has a route marked on her map; the other speaker -the Instruction Follower- has no route. The speakers are told that their goal is to reproduce the Instruction Giver's route on the Instruction Follower's map. The maps are not identical and the speakers are told this explicitly at the beginning of their first session. It is, however, up to them to discover how the two maps differ (HCRC Map Task Corpus 2008). 

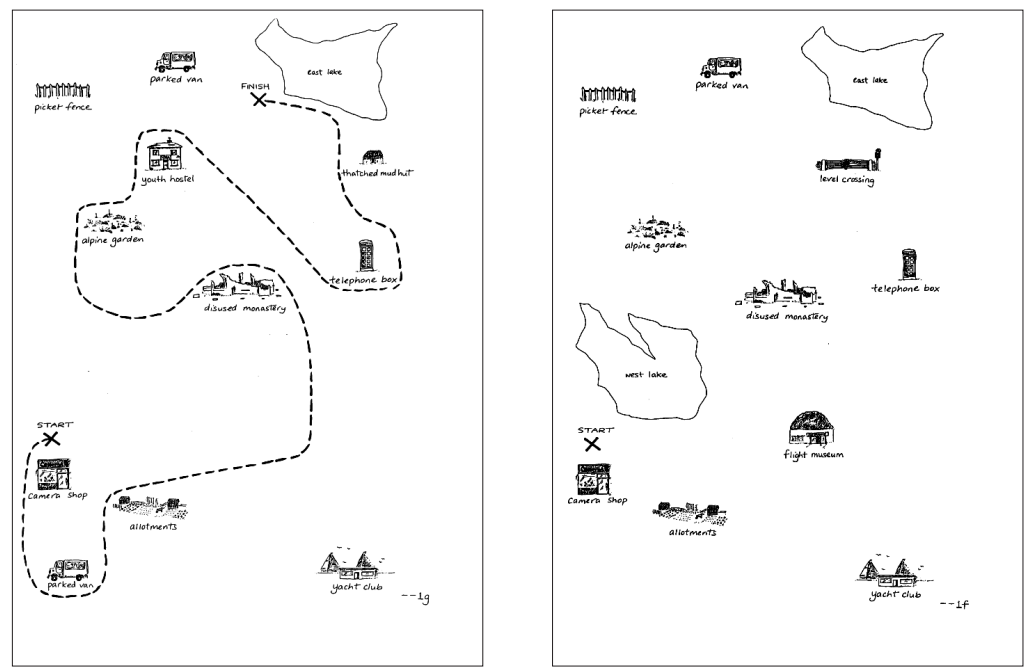

Figura 3. Mapas para la obtención del corpus Map task adaptado de HCRC Map task Corpus (2008): a la izquierda, el del Instruction Giver; a la derecha, el mapa del Instruction Follower

A continuación puede verse los mapas utilizados en este trabajo siguiendo las directrices del proyecto AMPER.
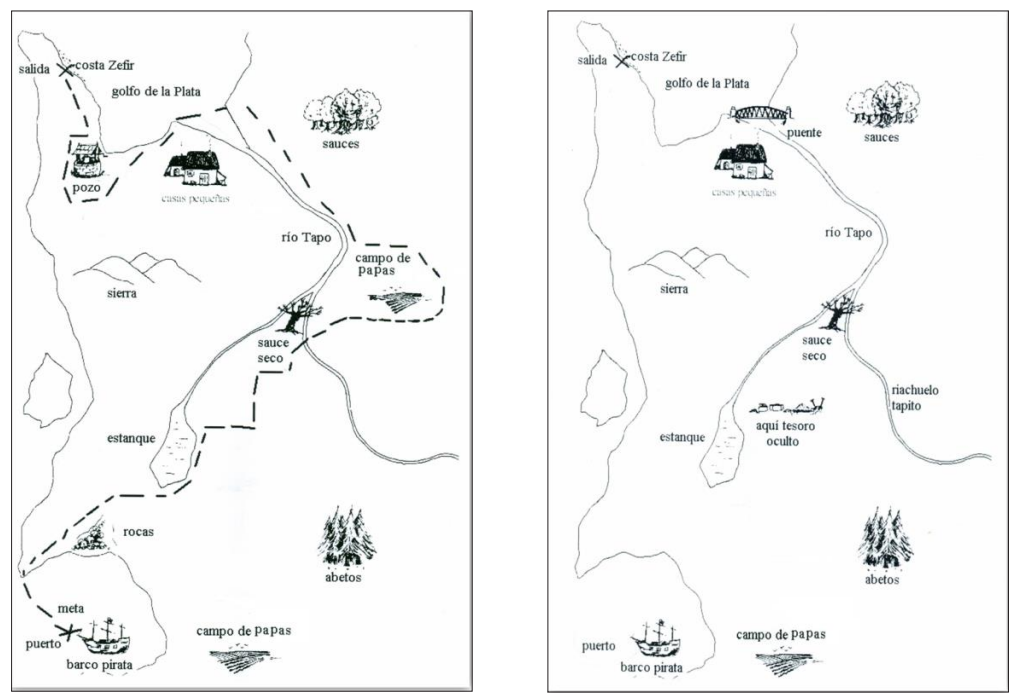

Figura 4. Mapas para la obtención del corpus Map task en este trabajo: a la izquierda, el del entrevistador; a la derecha, el mapa del informante 
Como puede deducirse, el corpus que se obtiene con este tipo de métodos es más espontáneo, pero a la vez controlado. De ahí que, considerando el grado de naturalidad, el habla analizada sea semiespontánea. Para los intereses del presente trabajo se han extraído 94 oraciones (66 declarativas y 28 interrogativas absolutas neutras) con esquema acentual final llano (tónica-átona [TA] o átona-tónica-átona [ATA]) que es el más frecuente en español (79,5\% según Quilis 1983). Véase un ejemplo en la figura 5 de la mujer de Aragua (en la Región Central).

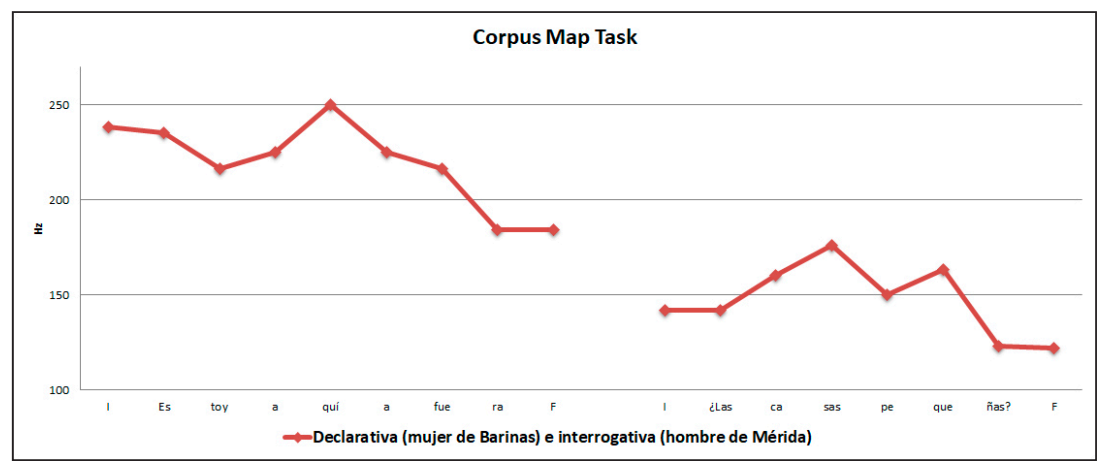

Figura 5. Ejemplos de declarativa e interrogativa extraídos del corpus Map task

Para una mayor naturalidad de las emisiones, se realizaron las encuestas en el lugar de residencia de los informantes. Las oraciones emitidas se grabaron con un ordenador portátil y la señal grabada fue tratada con el programa Goldwave Digital Audio Editor (versión 4.25) convirtiendo cada una de las frases en ficheros .wav, una vez eliminados los posibles ruidos y optimizada la señal resultante con una frecuencia de muestreo de $16 \mathrm{kHz}$. Los ficheros .wav se analizaron con el programa AMPER2006 (las subrutinas fueron diseñadas por el grupo Amper-Astur, López Bobo et al. 20077, a partir de las que originalmente había creado Antonio Romano 1995, de Amper-Italia). El análisis acústico tiene diferentes fases y cada frase es pasada por una serie de subrutinas. El punto de partida es el oscilograma de la frase en el que se hace una segmentación de las vocales que se analizan como bloques discretos asignándoles tres valores de F0: inicio, medio y final. De los tres parámetros implicados en la entonación, nos centramos en el estudio de la F0 en el centro del núcleo de la sílaba por ser la parte más estable. Posteriormente, el análisis fue supervisado con el programa Praat. 
Para llevar a cabo el estudio fonético-fonológico de la F0, nos hemos basado en el umbral psicoacústico de 1,5 St establecido por Rietveld y Gussenhoven (1985) y corroborado para el español por Pamies Bertrán et al. (2002). A su vez, la interpretación fonológica se ha realizado con el sistema ToBI (Tones and Break Indices) en el marco del modelo Métrico-Autosegmental (Pierrehumbert 1980), aplicada al español bajo la denominación de Spanish ToBI (Sp_ToBI); la primera propuesta de transcripción entonativa fue realizada por Beckman et al. (2002) y ha sido revisada posteriormente por varios autores (v. Hualde 2003; Fernández Planas y Martínez Celdrán 2003; Estebas y Prieto 2008; Prieto y Roseano, eds. 2010; Dorta et al. 2013a y en Dorta y Díaz 2018a). Véase en las siguientes tablas el sistema de invariantes y variantes de los acentos tonales y tonos de frontera extraídos de Dorta y Díaz (2018a) que se aplican en este trabajo.

\begin{tabular}{|c|c|}
\hline Estructura profunda & Estructura superficial \\
\hline Invariantes & Variantes \\
\hline \multirow{2}{*}{$/ \mathrm{L}^{*+\mathrm{H}} /$} & {$\left[\mathrm{L}^{*+\mathrm{H}}\right]$} \\
\hline & {$\left[\mathrm{L}^{*}+! \mathrm{H}\right]$} \\
\hline \multirow{3}{*}{$/ \mathrm{L}+\mathrm{H}^{*} /$} & {$\left[\mathrm{L}+\mathrm{H}^{*}\right]$} \\
\hline & {$\left[\mathrm{L}+{ }_{i} \mathrm{H}^{*}\right]$} \\
\hline & {$\left[\mathrm{L}+>\mathrm{H}^{*}\right]$} \\
\hline \multirow{4}{*}{$/ \mathrm{L}^{*} /$} & {$\left[\mathrm{L}^{*}\right]$} \\
\hline & {$\left[\mathrm{L}^{*+\mathrm{H}}\right]$} \\
\hline & {$\left[\mathrm{L}^{*}+! \mathrm{H}\right]$} \\
\hline & {$\left[! \mathrm{H}+\mathrm{L}^{*}\right]$} \\
\hline \multirow{5}{*}{$/ \mathrm{H}^{*} /$} & {$\left[\mathrm{H}^{*}\right]$} \\
\hline & {$\left[{ }_{i} \mathrm{H}^{*}\right]$} \\
\hline & {$\left[! \mathrm{H}^{*}\right]$} \\
\hline & {$\left[\mathrm{L}+\mathrm{H}^{*}\right]$} \\
\hline & {$\left[\mathrm{L}+{ }_{i} \mathrm{H}^{*}\right]$} \\
\hline$/ \mathrm{L}+! \mathrm{H}^{*} /$ & {$\left[\mathrm{L}+! \mathrm{H}^{*}\right]$} \\
\hline
\end{tabular}

Tabla 1. Representación de las invariantes y variantes de los acentos tonales 


\begin{tabular}{|c|c|}
\hline ESTRUCTURA PROFUNDA & ESTRUCTURA SUPERFICIAL \\
\hline Invariante fonológica & Variantes \\
\hline$/ \% \mathrm{H} /-/ \mathrm{H} \% /$ & {$[\mathrm{H} \%]$} \\
\hline \multirow{3}{*}{$/ \% \mathrm{M} /-/ \mathrm{M} \% /$} & {$[\% \mathrm{M}]-[\mathrm{M} \%]$} \\
\cline { 2 - 2 } & {$[\% \mathrm{MH}]-[\mathrm{MH} \%]$} \\
\cline { 2 - 2 } & {$[\% \mathrm{ML}]-[\mathrm{ML} \%]$} \\
\hline \multirow{3}{*}{$/ \% \mathrm{~L} /-/ \mathrm{L} \% /$} & {$[\% \mathrm{~L}]-[\mathrm{L} \%]$} \\
\cline { 2 - 2 } & {$\left[\mathrm{H}_{\mathrm{L}} \%\right]$} \\
\hline
\end{tabular}

Tabla 2. Representación de las invariantes y variantes de los tonos de frontera

\section{RESULTADOS}

\subsection{LAS DECLARATIVAS}

\subsubsection{Descripción general}

Las declarativas venezolanas analizadas se caracterizan en el final de manera diferente en mujeres y hombres: en las primeras se da un descenso hasta el final absoluto, en los segundos un ascenso antes del descenso final. Tal como se puede apreciar en la figura 6 , el tono medio general (TMG) distancia a los dos sexos (5,6 St) a favor del femenino (205 y $148 \mathrm{~Hz}$ en mujeres y hombres, respectivamente).

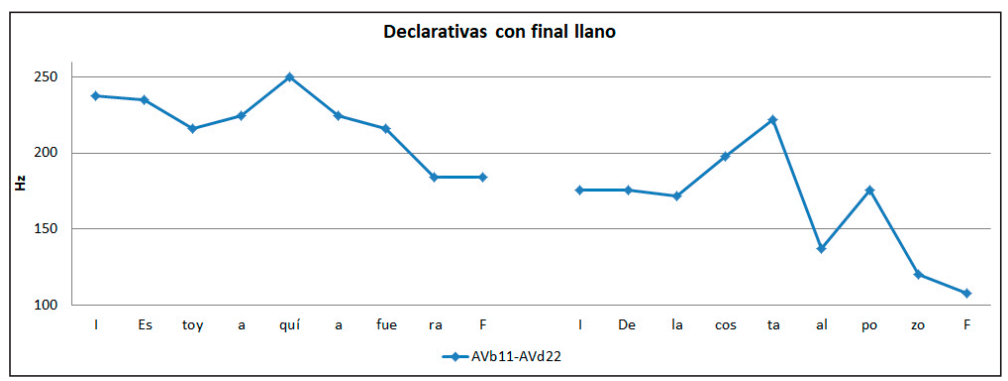

Figura 6. Declarativas con final llano emitidas por una mujer de Barinas (AVb11) y un hombre de Bolívar (AVd22) en un corpus Map task 


\subsubsection{Realizaciones de los tonos de frontera y de los acentos tonales}

$1^{\circ}$ ) Inicio TA (tónica-átona) y final llano ATA (átona-tónica-átona)

La figura 7 ilustra los contornos estilizados de las declarativas venezolanas ${ }^{8}$ con inicio tónico y final llano TA/ATA. En un porcentaje similar de casos $(47,1 \%$ y $45,5 \%)$, la frecuencia fundamental de mujeres y hombres asciende hasta un pico colocándose el inicio absoluto en torno a la media del informante (-0,2 y $0,7 \mathrm{St}$, respectivamente); en el 52,9\% restante de los casos de la voz femenina, la melodía registra un descenso desde el inicio situándose este significativamente por encima de la media $(2,1 \mathrm{St})$; en cambio, en el 54,5\% restante de los casos de los hombres, se da un ascenso continuo de la curva hasta el pico nuclear por lo que no hay pico inicial y el punto de partida se sitúa en la media del informante $(0,4 \mathrm{St})$.

En lo que respecta al núcleo entonativo, la diferente configuración también evidencia contrastes entre mujeres y hombres. En las primeras se da siempre un contorno final descendente de manera que el salto desde la pretónica a la tónica supera el umbral perceptivo $(-2,1 \mathrm{St})$, colocándose esta última vocal por debajo del TMG, aunque solo en las oraciones que no presentan pico inicial llega a superar las diferencias mínimas perceptibles (-0,7 St con pico y $-1,7 \mathrm{St}$ sin pico). En los hombres, en cambio, el contorno nuclear es mayoritariamente circunflejo $(84,6 \%)$ pues solo puntualmente es descendente en el hombre de Bolívar $\left(15,4 \%{ }^{9}\right)$. En el primero de estos finales, cuando las oraciones tienen pico inicial se produce un ascenso de la pretónica a la tónica $(1,8 \mathrm{St})$ dejando esta última ligeramente por encima del TMG (0,7 St), pero por debajo del valor del pico inicial (-1,6 St); en cambio, cuando las oraciones no tienen pico inicial el movimiento circunflejo abarca toda la frase por lo que, aunque el movimiento de la pretónica a la tónica sea imperceptible $(0,3 \mathrm{St})$, la tónica supera significativamente el TMG $(1,8$ St). Finalmente, en los dos sexos, el final absoluto medio de las curvas se sitúa significativamente por debajo del TMG (-3,6 y -2,7 St en mujeres y hombres, respectivamente).

\footnotetext{
8 I: inicio absoluto; P1: primer pico; T: tónica; PreN: pretónica nuclear; TN: tónica nuclear; PostN: postónica nuclear; F: final absoluto.

9 Este $15,4 \%$ de oraciones descendentes lo hemos excluido de este estudio para no distorsionar los resultados al tratarse de un patrón esporádico con diversa configuración (el $7,7 \%$ presenta pico inicial y el restante un descenso desde el inicio).
} 


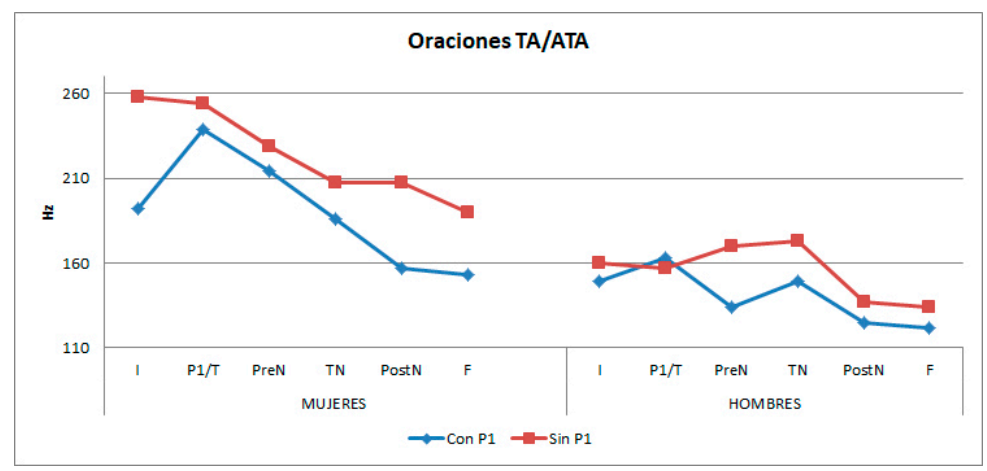

Figura 7. Estilización de los contornos entonativos de las frases declarativas con final llano que se inician con sílaba tónica

En la tabla 3 aparecen las variantes y los porcentajes correspondientes de los tonos de frontera inicial y final y de los acentos tonales inicial y final.

\begin{tabular}{|c|c|c|c|c|c|}
\hline Inicio-Final & Sexo & $\%$ Tono inicial & Acento inicial & Acento nuclear & Tono final \% \\
\hline \multirow[t]{2}{*}{ TA-ATA } & M & $\begin{array}{l}\% \mathrm{H} 47,1 \% \\
\% \mathrm{~L} \mathrm{5,9 \%} \\
\% \mathrm{M} 17,6 \% \\
\% \mathrm{MH} \mathrm{5,9 \%} \\
\% \mathrm{ML} \mathrm{23,5 \%}\end{array}$ & $\begin{array}{l}\mathrm{L}^{*}+\mathrm{H} 58,3 \% \\
\mathrm{H}^{*} 41,7 \%\end{array}$ & $\begin{array}{l}\mathrm{L}^{*}\left(/ \mathrm{L}^{*} /\right) 64,7 \% \\
\mathrm{H}^{*}\left(/ \mathrm{H}^{*} /\right) 35,3 \%\end{array}$ & $\begin{array}{l}\text { L\% 94,1\% } \\
\text { ML\% 5,9\% }\end{array}$ \\
\hline & $\mathrm{H}$ & $\begin{array}{l}\% \mathrm{H} \mathrm{9,1 \%} \\
\% \mathrm{~L} \mathrm{9,1 \%} \\
\% \mathrm{M} \mathrm{36,3 \%} \\
\% \mathrm{MH} \mathrm{18,2 \%} \\
\% \mathrm{ML} 27,3 \%\end{array}$ & $\begin{array}{l}\mathrm{L}^{*}+\mathrm{H} 60 \% \\
\mathrm{H}^{*} 30 \% \\
\mathrm{~L}+>\mathrm{H}^{*} 10 \%\end{array}$ & \begin{tabular}{|l}
$\mathrm{L}+\mathrm{H}^{*}\left(/ \mathrm{L}+\mathrm{H}^{*} /\right)$ \\
$27,3 \%$ \\
$\mathrm{~L}+\mathrm{H}^{*}\left(/ \mathrm{H}^{*} /\right) 9,1 \%$ \\
$\mathrm{H}^{*}\left(/ \mathrm{H}^{*} /\right) 45,4 \%$ \\
$! \mathrm{H}^{*}\left(/ \mathrm{H}^{*} /\right) 9,1 \%$ \\
$\mathrm{~L}^{*}\left(/ \mathrm{L}^{*} /\right) 9,1 \%$
\end{tabular} & $\mathrm{~L} \% 100 \%$ \\
\hline
\end{tabular}

Tabla 3. Realizaciones tonales en las frases declarativas con inicio tónico y final llano

Como puede verse, el tono de frontera inicial evidencia diferencias entre los dos sexos. Así, las mujeres inician las frases generalmente en un tono alto $([\% \mathrm{H}] 47,1 \%)$ o medio con distintas variantes $([\% \mathrm{MH}],[\% \mathrm{ML}]$ y [\%M] 47\%); los hombres, en cambio, mayoritariamente en torno a la media $([\% \mathrm{MH}],[\% \mathrm{ML}]$ y $[\% \mathrm{M}] 81,8 \%$ frente al tono alto con un $9,1 \%)$. 
Solo esporádicamente el inicio se coloca por debajo de la media ([\%L]) en los dos sexos (5,9\% en las mujeres y $9,1 \%$ en los hombres). Frente a esta variabilidad, en el primer acento, los dos sexos coinciden en el uso frecuente de un acento tonal bitonal con tónica baja $\left[\mathrm{L}^{*+\mathrm{H}}\right] \mathrm{y}$, en menor porcentaje, de uno monotonal con tónica alta $\left[\mathrm{H}^{*}\right]$.

En el núcleo entonativo las mujeres utilizan más frecuentemente un acento tonal con tónica baja $\left[\mathrm{L}^{*}\right](64,7 \%)$; no obstante, en un $35,3 \%$ la tónica queda alta $\left(\left[\mathrm{H}^{*}\right]\right)$ a pesar de que la trayectoria de la curva es descendente y concluye frecuentemente por debajo de la media del informante ([L\%]), debido a que la oración tiene un único acento o dos en situación de clash $\mathrm{y}$ al tratarse de inicio tónico no existen átonas precedentes que faciliten el descenso significativo de la F0. En los hombres, el final circunflejo favorece la aparición de diferentes variantes nucleares dependiendo de las variables siguientes: a) si existe valle previo al pico nuclear $(36,4 \%)$ sea relevante la subida (/L+H*/27,3\%) o no $\left(/ \mathrm{H}^{*} / 9,1 \%\right)$; b) de si el último pico presenta escalonamiento descendente ([! $\left.\left.\mathrm{H}^{*}\right] 9,1 \%\right)$; c) de si, fundamentalmente, se da una subida continua generalmente desde el inicio dejando una tónica alta $\left(\left[\mathrm{H}^{*}\right] 45,4 \%\right)$, o d) de si el ascenso del pico hasta la última tónica no alcanza el semitono y medio $(9,1 \%)$ y, por ello, etiquetamos $/ \mathrm{L}^{*} /$ como en las mujeres. En todo caso, en los hombres, como sucedía mayoritariamente en las mujeres, el final se sitúa significativamente por debajo del TM ([L\%], $100 \%)$.

$2^{\circ}$ ) Inicio AT-ATA (átona-tónica o átona-tónica-átona) y final llano ATA (átona-tónica-átona)

Los inicios átonos de las declarativas con final llano de la figura 8 muestran los mismos contornos que vimos en los tónicos. No obstante, en este caso la mayoría de las oraciones de las mujeres tienen pico en la frontera inicial $(83,3 \%)$, manteniéndose el inicio absoluto de las curvas en torno a la media $(0,3 \mathrm{St})$; cuando no hay pico inicial $(16,7 \%)$, el inicio se coloca significativamente por encima de la media $(2 \mathrm{St})$. En los hombres el patrón más general vuelve a ser el circunflejo $(88,9 \%)$ y el descendente sigue apareciendo puntualmente en Bolívar (11,1\%). En el primero, como en las mujeres, son más frecuentes las oraciones que se inician con pico $(81,3 \%)$ que las que tienen un ascenso continuo hasta el pico nuclear (18,7\%); en ambos casos, las curvas se inician en el TMG $(0,1 \mathrm{St}$ y $-0,9 \mathrm{St}$, respectivamente).

En el núcleo entonativo, al contrario que en las oraciones que comenzaban por inicio tónico, el descenso que se produce desde la pretónica a la tónica es relevante en las oraciones sin pico de las mujeres $(-1,7 \mathrm{St})$, pero en las que tienen pico no se supera el umbral diferencial $(-1,4 \mathrm{St})$; no obstante, en los dos casos la tónica se sitúa en el TM (-0,3 St con pico y 0 St sin pico). 
En los hombres, como veíamos con los inicios tónicos, el ascenso desde la pretónica a la tónica es relevante $(1,6 \mathrm{St})$ en las oraciones que se inician con pico e imperceptible $(0,7 \mathrm{St})$ en las que se da ascenso continuo desde el inicio; la relación de la tónica con el tono medio, en cambio, presenta diferencias relevantes puesto que si bien los dos tipos de oraciones quedan por encima del TMG, las primeras lo hacen solo ligeramente $(1 \mathrm{St})^{10}$ y las segundas de manera significativa $(2,8 \mathrm{St})$. En todos los casos, el final medio se sitúa por debajo del TMG (-3,6 y -3 St en mujeres y hombres, respectivamente).

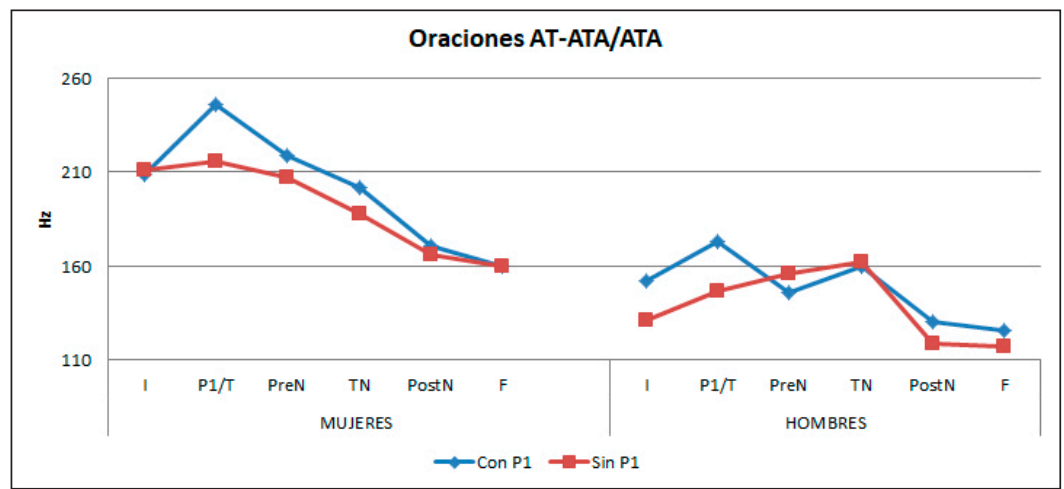

Figura 8. Estilización de los contornos entonativos de las frases declarativas con final llano que se inician con sílaba átona

Acorde con la descripción de los párrafos precedentes podemos ver en la Tabla 4 las realizaciones de los tonos de frontera y de los acentos tonales inicial y final.

10 El valor de la tónica nuclear donde se sitúa el pico final es inferior al alcanzado por el del primer pico aunque las diferencias no alcanzan el umbral $(1,4 \mathrm{St})$. 


\begin{tabular}{|c|c|c|c|c|c|}
\hline \begin{tabular}{|l|} 
Inicio-Final \\
\end{tabular} & Sexo & $\%$ Tono inicial & Acento inicial & Acento nuclear & Tono final \% \\
\hline \multirow[t]{2}{*}{ AT-ATA/AT } & $\mathrm{M}$ & $\begin{array}{l}\% \mathrm{H} 38,9 \% \\
\% \text { L } 11,1 \% \\
\% \mathrm{M} 16,7 \% \\
\% \mathrm{MH} 22,2 \% \\
\% \mathrm{ML} 11,1 \%\end{array}$ & $\begin{array}{l}\mathrm{L} * \mathrm{H} 43,8 \% \\
\mathrm{H} * 25 \% \\
\mathrm{~L}+>\mathrm{H}^{*} 6,2 \% \\
\mathrm{~L}+\mathrm{H}^{*} 25 \%\end{array}$ & $\mathrm{~L} *(/ \mathrm{L} * /) 100 \%$ & L\% 100\% \\
\hline & $\mathrm{H}$ & $\begin{array}{l}\% \text { M 43,8\% } \\
\% \text { MH 6,2\% } \\
\% \text { ML 31,3\% } \\
\% \text { L 12,5\% } \\
\% \text { H 6,2\% }\end{array}$ & $\begin{array}{l}\mathrm{L} * \mathrm{H} 50 \% \\
\mathrm{H}^{*} 6,3 \% \\
\mathrm{~L}+>\mathrm{H}^{*} 25 \% \\
\mathrm{~L}+\mathrm{H}^{*} 18,7 \%\end{array}$ & $\begin{array}{l}\mathrm{L}+\mathrm{H}^{*}\left(/ \mathrm{L}+\mathrm{H}^{*} /\right) 31,3 \% \\
\mathrm{~L}+\mathrm{H}^{*}\left(/ \mathrm{H}^{*} /\right) 12,5 \% \\
\mathrm{~L}+! \mathrm{H}^{*}\left(/ \mathrm{L}+\mathrm{H}^{*} /\right) 12,5 \% \\
\mathrm{H}^{*}\left(/ \mathrm{H}^{*} /\right) 18,7 \% \\
! \mathrm{H}^{*}\left(/ \mathrm{H}^{*} /\right) 6,3 \% \\
\mathrm{~L}^{*}\left(/ \mathrm{L}^{*} /\right) 18,7 \%\end{array}$ & $\begin{array}{l}\mathrm{L} \% 87,5 \% \\
\mathrm{ML} \% 12,5 \%\end{array}$ \\
\hline
\end{tabular}

Tabla 4. Realizaciones tonales en las frases declarativas con inicio átono y final llano

Lo más general en los hombres y menos significativamente en las mujeres es que el tono de frontera inicial se coloque en la media del informante ([\%MH], [\%ML] y [\%M] 81,3\% y 50\%, respectivamente); en las mujeres es destacable el porcentaje del acento monotonal [\%H] (38,9\%). En lo que respecta al acento inicial, como en los inicios tónicos, es más habitual el bitonal con tónica baja $\left[\mathrm{L}^{*}+\mathrm{H}\right]$ en los dos sexos $(43,8 \%$ en las mujeres y $50 \%$ en los hombres); en el resto de los casos, la tónica queda alta repartiéndose el porcentaje entre los alotonos $\left[\mathrm{H}^{*}\right],\left[\mathrm{L}+\mathrm{H}^{*}\right]$ y $\left[\mathrm{L}+>\mathrm{H}^{*}\right]$. Por último, el núcleo entonativo presenta nuevamente las mayores diferencias entre mujeres y hombres por la presencia de los dos contornos descritos anteriormente:

a) En las primeras se da el descenso hasta el final absoluto quedando la tónica baja y el final significativamente por debajo del TM ([L* L\%], 100\%).

b) En los hombres, se da un movimiento ascendente-descendente caracterizado por diferentes esquemas nucleares entre los que destacan las diversas variantes del bitonal / $\mathrm{L}+\mathrm{H}^{*} /\left(\left[\mathrm{L}+\mathrm{H}^{*}\right],\left[\mathrm{L}+\mathrm{H}^{*}\right] 43,8 \%\right)$ frente al monotonal $/ \mathrm{H}^{*} /\left(\left[\mathrm{L}+\mathrm{H}^{*}\right],\left[\mathrm{H}^{*}\right]\right.$ y $\left.\left[! \mathrm{H}^{*}\right] 37,5 \%\right)$. Los casos en que el ascenso hasta el pico final no son relevantes desde el punto de vista perceptivo son muy bajos (/L*/18,7\%). 


\subsection{LAS INTERROGATIVAS}

\subsubsection{Descripción general}

En esta modalidad, hemos registrado, de manera general en los dos sexos, el final circunflejo. No obstante, se da también, aunque con menor frecuencia, un final descendente prácticamente en su totalidad en la mujer de Barinas. En la figura 9 ilustramos el contorno circunflejo más general; se puede apreciar que el TMG distancia a las mujeres de los hombres por encima del umbral considerado (216 y $170 \mathrm{~Hz}$, respectivamente; 4,1 St).

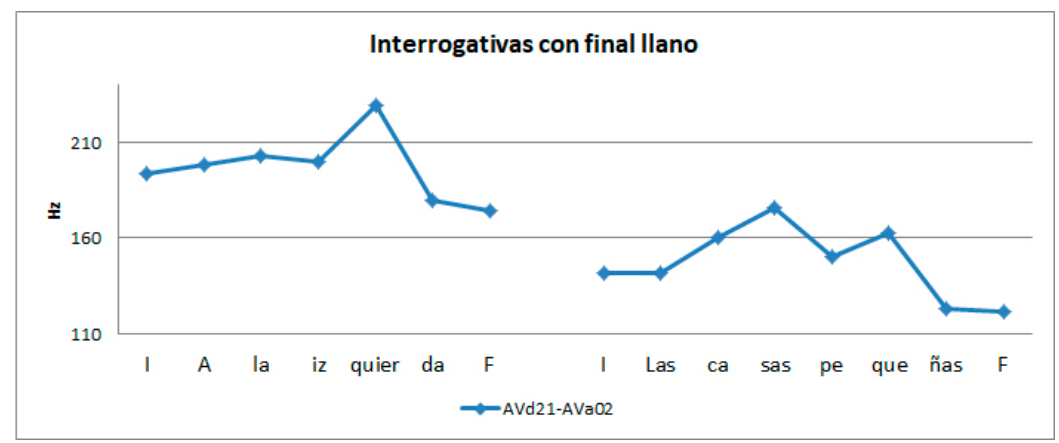

Figura 9. Interrogativas con final llano emitidas por una mujer de Bolívar (AVd21) y un hombre de Mérida (AVa02) en un corpus Map task

\subsubsection{Realizaciones de los tonos de frontera y de los acentos tonales}

$1^{\circ}$ ) Inicio TA (tónica-átona) y final llano ATA (átona-tónica-átona)

Como puede verse en la figura 10 , solo hemos registrado oraciones con inicio tónico en las mujeres. En todos los casos, la estructura del núcleo entonativo es circunfleja de manera que considerando la distancia desde la pretónica a la tónica nuclear y de esta última respecto del TMG, el resultado es un valor relevante perceptivamente $(2,7 \mathrm{St})$; el descenso final brusco $(-4,2 \mathrm{St})$ deja el término de las curvas significativamente por debajo del TMG. 


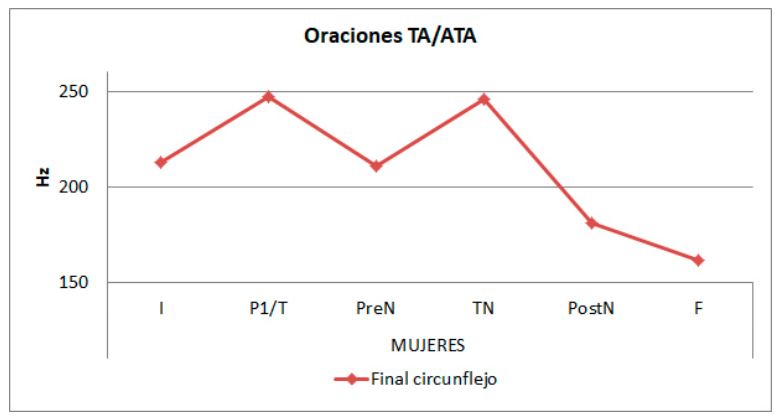

Figura 10. Estilización de los contornos entonativos de las frases interrogativas que se inician con sílaba tónica

Si observamos el tono de frontera inicial podemos ver en la tabla 5 que la mitad de las oraciones tienen su punto de partida por encima del TM $([\% \mathrm{H}])$ y la otra mitad por debajo $([\% \mathrm{~L}])$. En el acento inicial, asimismo, las variantes se reparten al $50 \%$ : tónica baja y acenso posterior $\left(\left[\mathrm{L}^{*}+\mathrm{H}\right]\right)$ y tónica alta y pico posterior, es decir, acento desplazado $\left(\left[\mathrm{L}+>\mathrm{H}^{*}\right]\right)$. En lo que respecta al acento nuclear y el tono de frontera final, el contorno circunflejo más frecuente es el que presenta acento monotonal alto seguido del tono de frontera bajo $\left(/ \mathrm{H}^{*} \mathrm{~L} \% /, 83,3 \%\right)$ con diferentes variantes $\left(\left[\mathrm{L}+\mathrm{H}^{*}\right],\left[\mathrm{L}+\mathrm{H}^{*}\right]\right.$, $\left[\mathrm{H}^{*}\right]$ y $\left.\left[\mathrm{iH}^{*}\right]\right)$; con menor frecuencia encontramos un ascenso significativo de la F0 hasta la tónica nuclear con posterior descenso hasta el final $\left(/ \mathrm{L}+\mathrm{H}^{*}\right.$ $\mathrm{L} \% /, 16,7 \%)$ con una única realización ([L+H* L\%]).

\begin{tabular}{|c|c|c|c|c|c|}
\hline Inicio-Final & Sexo & $\%$ Tono inicial & Acento inicial & Acento nuclear & Tono final $\%$ \\
\hline \multirow{6}{*}{ TA-ATA } & \multirow{5}{*}{ M } & $\% \mathrm{H} 50 \%$ & $\mathrm{~L}^{*}+\mathrm{H} 50 \%$ & $\mathrm{~L}+\mathrm{H}^{*}\left(/ \mathrm{L}+\mathrm{H}^{*} /\right) 16,7 \%$ & $\mathrm{~L} \% 100 \%$ \\
\hline & & $\%$ L $50 \%$ & $\mathrm{~L}+>\mathrm{H}^{*} 50 \%$ & $\mathrm{~L}+\mathrm{H}^{*}\left(/ \mathrm{H}^{*} /\right) 16,7 \%$ & \\
\hline & & & & $\mathrm{L}+! \mathrm{H}^{*}\left(/ \mathrm{H}^{*} /\right) \quad 16,7 \%$ & \\
\hline & & & & $H^{*}\left(/ H^{*} /\right) 33,2 \%$ & \\
\hline & & & & ${ }_{i} \mathrm{H}^{*}\left(/ \mathrm{H}^{*} /\right)$ 16,7\% & \\
\hline & $\mathrm{H}$ & - & - & - & - \\
\hline
\end{tabular}

Tabla 5. Realizaciones tonales en las frases interrogativas con inicio tónico y final llano

$2^{\circ}$ ) Inicio AT-ATA (átona-tónica o átona-tónica-átona) y final llano ATA (átona-tónica-átona) 
Como puede verse en la figura 11, si bien en los hombres se da en todos los casos un final circunflejo cuando el inicio es átono, en las mujeres el comportamiento tonal evidencia la existencia de dos patrones nucleares de los cuales el circunflejo es el más frecuente $(71,4 \%)$ como en el otro sexo. El segundo patrón $(28,6 \%)$ no presenta pico destacado antes del descenso final por lo que es descendente, sobre todo en la mujer de Barinas $(21,4 \%)$ y puntualmente en la de Aragua (7,1\%). En el corpus formal (v. apartado 1) pudimos ver que la primera informante se caracterizaba por la entonación descendente de sus interrogativas; en el que hemos analizado ahora, en cambio, se pone de relieve que junto al patrón descendente $(50 \%)$ es posible la entonación circunfleja (50\%). Por otra parte, tal como se ha visto para el corpus formal (Díaz y Dorta 2018 b), si comparamos una interrogativa descendente con una circunfleja la diferencia en el TM entre ambas es imperceptible ( $0,2 \mathrm{St})$; sin embargo, al comparar las interrogativas descendentes con las declarativas descendentes femeninas con inicio átono se puede comprobar que la diferencia a favor de las primeras es relevante $(2,2 \mathrm{St}) ;$ por tanto, la mayor altura tonal de una frente a la otra es un rasgo que permite diferenciar las dos modalidades.

Por otro lado, con independencia del patrón final, las curvas se inician en torno a la media en las mujeres (-01 St) y significativamente por debajo en los hombres $(-1,9 \mathrm{St})$. No obstante, el núcleo según el contorno final varía:

- En el descendente se produce un descenso irrelevante de la pretónica a la tónica $(-1,2 \mathrm{St})$, aunque esta última se coloca por debajo del TMG (-2,2 St); ya en el final absoluto la distancia respecto de la media supera bastante la media $(-4,4 \mathrm{St})$.

- En el circunflejo se da un ascenso medio relevante desde el punto de vista perceptivo en el salto de la pretónica a la tónica $(1,7 \mathrm{St}$ en mujeres y 2,8 St en hombres) que deja a esta última vocal por encima del TMG (2,3 St en mujeres y 2,5 St en hombres) y por debajo al final de este (-3,1 St en mujeres y $-2,5 \mathrm{St}$ en hombres). 


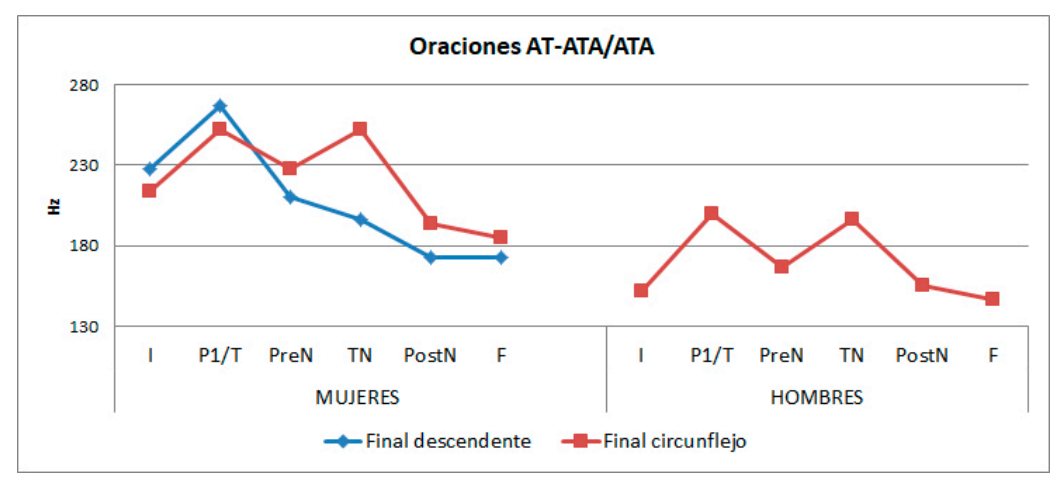

Figura 11. Estilización de los contornos entonativos de las frases interrogativas que se inician con sílaba átona

Teniendo en cuenta la descripción realizada, observamos en la tabla 6 que las mujeres usan generalmente $(92,9 \%)$ un tono medio inicial con diferentes variantes $([\% \mathrm{MH}],[\% \mathrm{ML}]$ y $[\% \mathrm{M}])$, frente a los hombres que emplean uno bajo ([\%L], 62,5\%). En el acento inicial se dan, asimismo, discrepancias entre los dos sexos: mientras las mujeres emplean frecuentemente el acento bitonal con tónica baja $\left(\left[\mathrm{L}^{*}+\mathrm{H}\right], 45,4 \%\right)$, los hombres utilizan mayoritariamente el esquema con desplazamiento del pico ([L+> $\left.\left.\mathrm{H}^{*}\right], 75 \%\right)$, esquema este que en las mujeres alcanza el peor porcentaje $(18,2 \%)$.

En el núcleo, el patrón circunflejo más general (el más frecuente) es el que presenta un acento nuclear alto $/ \mathrm{H}^{*} /(71,4 \%$ en mujeres y $75 \%$ en hombres $)$ con distintas variantes $\left(\left[\mathrm{L}+\mathrm{H}^{*}\right],\left[\mathrm{H}^{*}\right]\right.$ y $\left[\mathrm{L}+{ }_{i} \mathrm{H}^{*}\right]$ o $\left.\left[{ }_{i} \mathrm{H}^{*}\right]\right)$; el descenso a partir del pico deja el final por debajo del TM (92,9\% y 87,5\%), salvo excepciones en que queda en torno al tono medio. Los casos en que las mujeres no usan el patrón circunflejo sino el descendente ([L* L\%], 28,6\%) son, como se ha dicho, más escasos. 


\begin{tabular}{|c|c|c|c|c|c|}
\hline Inicio-final & Sexo & $\%$ Tono inicial & Acento inicial & Acento nuclear & Tono final \% \\
\hline \multirow{10}{*}{ AT-ATA/AT } & \multirow[t]{6}{*}{$\mathrm{M}$} & $\% \mathrm{~L} 7,1 \%$ & $\mathrm{~L} *+\mathrm{H} 45,4 \%$ & $\mathrm{~L}+\mathrm{H}^{*} \quad\left(/ \mathrm{L}+\mathrm{H}^{*} /\right)$ & $\mathrm{L} \% 92,9 \%$ \\
\hline & & $\% \mathrm{M} 28,6 \%$ & $\mathrm{H}^{*} 36,4 \%$ & $28,6 \%$ & ML \% 7,1\% \\
\hline & & $\%$ MH 42,9\% & $\mathrm{L}+>\mathrm{H}^{*} 18,2 \%$ & $\mathrm{~L}+\mathrm{H}^{*}\left(/ \mathrm{H}^{*} /\right) 14,3 \%$ & \\
\hline & & $\%$ ML $21,4 \%$ & & $\mathrm{~L}+{ }_{i} \mathrm{H}^{*}\left(/ \mathrm{H}^{*} /\right) 7,1 \%$ & \\
\hline & & & & $\mathrm{H}^{*}\left(/ \mathrm{H}^{*} /\right) 21,4 \%$ & \\
\hline & & & & $\mathrm{L} *(/ \mathrm{L} * /) 28,6 \%$ & \\
\hline & \multirow[t]{4}{*}{$\mathrm{H}$} & $\%$ M $25 \%$ & $\mathrm{~L}+>\mathrm{H}^{*} 75 \%$ & $\mathrm{~L}+\mathrm{H}^{*}\left(/ \mathrm{L}+\mathrm{H}^{*} /\right) 25 \%$ & $\mathrm{~L} \% 87,5 \%$ \\
\hline & & $\%$ ML $12,5 \%$ & $\mathrm{~L}+\mathrm{H}^{*} 25 \%$ & $\mathrm{~L}+\mathrm{H}^{*}\left(/ \mathrm{H}^{*} /\right) 12,5 \%$ & ML\% $12,5 \%$ \\
\hline & & $\% \mathrm{~L} 62,5 \%$ & & $\mathrm{H}^{*}\left(/ \mathrm{H}^{*} /\right) 50 \%$ & \\
\hline & & & & ¡H* $\left(/ \mathrm{H}^{*} /\right)$ 12,5\% & \\
\hline
\end{tabular}

Tabla 6. Realizaciones tonales en las frases interrogativas con inicio átono y final llano

\subsection{INVARIANTES Y VARIANTES}

En este apartado consideraremos las invariantes y variantes más frecuentes en el corpus analizado. Así, si comparamos los inicios tónicos (TA) y átonos (AT-ATA) en las declarativas a partir de los datos ilustrados en la tabla 7, podemos ver que en los hombres el inicio se coloca mayoritariamente en la media del informante $(/ \% \mathrm{M} /)$, mientras que en las mujeres esto sucede solo en los inicios átonos, pues en los tónicos alterna con un tono alto $(/ \% \mathrm{H} /)$, debido en parte a que en esta modalidad las oraciones que se inician sin pico y a una frecuencia alta son más elevadas.

En el acento inicial, en cambio, ambos sexos coinciden en utilizar mayoritariamente un acento bitonal con tono bajo seguido de tono alto $\left(/ \mathrm{L}^{*}+\mathrm{H} /\right)$ aunque los porcentajes de aparición de la invariante en ambos sexos son más elevados cuando el inicio es tónico.

En el acento nuclear y en el tono de frontera final, las mujeres utilizan un tono bajo $\left(/ \mathrm{L}^{*} \mathrm{~L} \% /\right)$, sobre todo si el inicio es átono; en los hombres la invariante más frecuente para el final circunflejo es el acento monotonal alto $\left(/ \mathrm{H}^{*} /\right)$ seguido del tono de frontera bajo $(/ \mathrm{L} \% /)$, máxime si las oraciones inician con vocal tónica. 


\begin{tabular}{|c|c|c|c|c|c|c|c|c|c|}
\hline \multicolumn{10}{|c|}{ DECLARATIVAS } \\
\hline \multirow[t]{2}{*}{ Inicio-Final } & \multirow[t]{2}{*}{ Sexo } & \multicolumn{2}{|c|}{$\%$ Tono inicial } & \multicolumn{2}{|c|}{ Acento inicial } & \multicolumn{2}{|c|}{ Acento nuclear } & \multicolumn{2}{|c|}{ Tono final \% } \\
\hline & & Inv. & Var. & Inv. & Var. & Inv. & Var. & Inv. & Var. \\
\hline \multirow[t]{2}{*}{ TA-ATA } & $M$ & $\begin{array}{l}\% \mathrm{H} 47,1 \% \\
\% \mathrm{M} 47 \%\end{array}$ & $\begin{array}{l}\% \mathrm{H} \\
\% \mathrm{ML} \\
\end{array}$ & $\begin{array}{l}\mathrm{L}^{*+\mathrm{H}} \\
58,3 \%\end{array}$ & $\mathrm{~L}^{*+\mathrm{H}}$ & $\mathrm{L}^{*} 64,7 \%$ & $\mathrm{~L}^{*}$ & $\begin{array}{l}\mathrm{L} \% \\
94,1 \% \\
\end{array}$ & $\mathrm{~L} \%$ \\
\hline & $\mathrm{H}$ & $\% \mathrm{M} 81,8 \%$ & $\% \mathrm{M}$ & $\begin{array}{l}\mathrm{L}^{*+\mathrm{H}} \\
60 \% \\
\end{array}$ & $\mathrm{~L}^{*}+\mathrm{H}$ & $\mathrm{H}^{*} 63,6 \%$ & $\mathrm{H}^{*}$ & L\% 100\% & $\mathrm{L} \%$ \\
\hline \multirow{2}{*}{ AT-ATA/ATA } & $\mathrm{M}$ & $\% \mathrm{M} \mathrm{50 \%}$ & $\% \mathrm{MH}$ & $\begin{array}{l}\mathrm{L}^{*+\mathrm{H}} \\
43,8 \%\end{array}$ & $\mathrm{~L} *+\mathrm{H}$ & $L^{*} 100 \%$ & $\mathrm{~L}^{*}$ & L\% 100\% & $\mathrm{L} \%$ \\
\hline & $\mathrm{H}$ & $\% \mathrm{M} 81,3 \%$ & $\% \mathrm{M}$ & $\begin{array}{l}\mathrm{L}^{*+\mathrm{H}} \\
50 \%\end{array}$ & $\mathrm{~L}^{*}+\mathrm{H}$ & $\mathrm{L}+\mathrm{H}^{*} 43,8 \%$ & $\mathrm{~L}+\mathrm{H}^{*}$ & L\% $100 \%$ & $\mathrm{~L} \%$ \\
\hline
\end{tabular}

Tabla 7. Acentos tonales y variantes más frecuentes en las frases declarativas con final llano e inicio átono y tónico

En lo que respecta a las interrogativas, en la tabla 8 puede verse que cuando los inicios son tónicos, las mujeres alternan el tono de frontera alto $(/ \% \mathrm{H} /)$ con el bajo ( $/ \% \mathrm{~L} /)$; en cambio, si el inicio es átono, el tono es medio $(/ \% \mathrm{M} /)$. En los hombres, como hemos dicho, no se registraron inicios tónicos y en los átonos el tono de frontera frecuente es bajo $(/ \% \mathrm{~L} /)$. En relación con el acento inicial, si el inicio es tónico en las mujeres alterna el esquema $/ \mathrm{L}^{*}+\mathrm{H} /$ con $/ \mathrm{L}+\mathrm{H}^{*} /$; si el inicio es átono se da el bitonal $/ \mathrm{L}^{*}+\mathrm{H} /$ en las mujeres y / $\mathrm{L}+\mathrm{H}^{*} /$ en los hombres. Finalmente, el acento nuclear y el tono de frontera final con más porcentaje de aparición es $/ \mathrm{H}^{*} \mathrm{~L} \% /$.

\begin{tabular}{|c|c|c|c|c|c|c|c|c|c|}
\hline \multicolumn{10}{|c|}{ INTERROGATIVAS } \\
\hline \multirow[t]{2}{*}{ Inicio-final } & \multirow[t]{2}{*}{ Sexo } & \multicolumn{2}{|c|}{$\%$ Tono inicial } & \multicolumn{2}{|c|}{ Acento inicial } & \multicolumn{2}{|c|}{ Acento nuclear } & \multicolumn{2}{|c|}{ Tono final \% } \\
\hline & & Inv. & Var. & Inv. & Var. & Inv. & Var. & Inv. & Var. \\
\hline \multirow[t]{2}{*}{ TA-ATA } & M & $\begin{array}{l}\% \mathrm{H} 50 \% \\
\% \mathrm{~L} 50 \%\end{array}$ & $\begin{array}{l}\% \mathrm{H} \\
\% \mathrm{~L}\end{array}$ & $\begin{array}{l}\mathrm{L}^{*}+\mathrm{H} \\
50 \% \\
\mathrm{~L}+\mathrm{H}^{*} \\
50 \% \\
\end{array}$ & $\begin{array}{l}\mathrm{L} *+\mathrm{H} \\
\mathrm{L}+>\mathrm{H}^{*}\end{array}$ & $\mathrm{H}^{*} 83,3 \%$ & $\mathrm{H}^{*}$ & L\% $100 \%$ & $\mathrm{~L} \%$ \\
\hline & $\mathrm{H}$ & - & - & - & - & - & - & - & - \\
\hline \multirow[t]{2}{*}{ AT-ATA/ATA } & $\mathrm{M}$ & $\% \mathrm{M} 92,9 \%$ & $\% \mathrm{MH}$ & $\begin{array}{l}*+\mathrm{H} \\
45,4 \%\end{array}$ & $\mathrm{~L}^{*}+\mathrm{H}$ & $\mathrm{H}^{*} 42,8 \%$ & $\mathrm{H}^{*}$ & $\begin{array}{l}\mathrm{L} \% \\
92,9 \% \\
\end{array}$ & $\mathrm{~L} \%$ \\
\hline & $\mathrm{H}$ & $\% \mathrm{~L} 62,5 \%$ & $\% \mathrm{~L}$ & $\begin{array}{l}\mathrm{L}+\mathrm{H}^{*} \\
100 \%\end{array}$ & $\mathrm{~L}+>\mathrm{H}^{*}$ & $\mathrm{H}^{*} 75 \%$ & $\mathrm{H}^{*}$ & $\begin{array}{l}\mathrm{L} \% \\
87,5 \%\end{array}$ & $\mathrm{~L} \%$ \\
\hline
\end{tabular}

Tabla 8. Acentos tonales y variantes más frecuentes en las frases interrogativas con final llano e inicio átono y tónico

En la tabla 9 aparecen solo las invariantes con mayor frecuencia de uso. Como puede verse, en el tono de frontera inicial y en el acento inicial de las interrogativas hay mayor diversidad que en las declarativas; con todo, lo más destacable es que en el acento inicial $/ \mathrm{L}^{*}+\mathrm{H} /$ aparece en las dos 
modalidades aunque en las interrogativas junto a este acento bitonal con tónica baja alterna $/ \mathrm{L}+\mathrm{H}^{*} /$ con tónica alta.

\begin{tabular}{|c|c|c|c|c|c|c|c|c|c|}
\hline \multicolumn{10}{|c|}{ DECLARATIVAS (D) vs. INTERROGATIVAS (I) } \\
\hline \multirow[t]{2}{*}{ Inicio-final } & \multirow[t]{2}{*}{ Sexo } & \multicolumn{2}{|c|}{$\%$ Tono inicial } & \multicolumn{2}{|c|}{ Acento inicial } & \multicolumn{2}{|c|}{ Acento nuclear } & \multicolumn{2}{|c|}{ Tono final \% } \\
\hline & & $\mathrm{D}$ & I & $\mathrm{D}$ & I & $\mathrm{D}$ & I & $\mathrm{D}$ & I \\
\hline \multirow{3}{*}{ TA-ATA } & M & $\% \mathrm{H}$ & $\% \mathrm{H}$ & $\mathrm{L}^{*}+\mathrm{H}$ & $\mathrm{L}^{*}+\mathrm{H}$ & $\mathrm{L}^{*}$ & $\mathrm{H}^{*}$ & $\mathrm{~L} \%$ & $\mathrm{~L} \%$ \\
\hline & & $\% \mathrm{M}$ & $\% \mathrm{~L}$ & & $\mathrm{~L}+\mathrm{H}^{*}$ & & & & \\
\hline & $\mathrm{H}$ & $\% \mathrm{M}$ & - & $\mathrm{L} *+\mathrm{H}$ & - & $\mathrm{H}^{*}$ & - & $\mathrm{L} \%$ & - \\
\hline \multirow[b]{2}{*}{ AT-ATA/ATA } & M & $\% \mathrm{M}$ & $\% \mathrm{M}$ & $\mathrm{L}^{*}+\mathrm{H}$ & $\mathrm{L}^{*}+\mathrm{H}$ & $\mathrm{L}^{*}$ & $\mathrm{H}^{*}$ & $\mathrm{~L} \%$ & $\mathrm{~L} \%$ \\
\hline & $\mathrm{H}$ & $\% \mathrm{M}$ & $\% \mathrm{~L}$ & $\mathrm{~L}^{*+\mathrm{H}}$ & $\mathrm{L}+\mathrm{H}^{*}$ & $\mathrm{~L}+\mathrm{H}^{*}$ & $\mathrm{H}^{*}$ & $\mathrm{~L} \%$ & $\mathrm{~L} \%$ \\
\hline
\end{tabular}

Tabla 9. Distinción entre declarativas e interrogativas según los tonos de frontera y acentos tonales más frecuentes

El acento nuclear y el tono de frontera final, por su parte, describen un contraste entre declarativas e interrogativas en el caso de las mujeres: / $\mathrm{L}^{*}$ $\mathrm{L} \% / v s . / \mathrm{H}^{*} \mathrm{~L} \% /$, es decir, patrón descendente vs. alto-descendente. En los hombres, en cambio, se registra este último en las dos modalidades, aunque en las declarativas con inicio átono puede precederle un valle (/L+H* $\mathrm{L} \% /)$. Por tanto, lo más relevante es que en el tramo final de las curvas no se podría distinguir la modalidad en todos los casos a partir del etiquetaje aplicado. Existen, no obstante, otras evidencias fonéticas para diferenciar declarativas e interrogativas. Así, si consideramos la distancia media del pico nuclear de declarativas $(161 \mathrm{~Hz})$ e interrogativas $(196 \mathrm{~Hz})$, podemos comprobar (figura 12) que la distancia es significativa (3,4 St), favoreciendo, como en las mujeres, la mayor altura de las interrogativas. Por ello, se hace necesario ampliar los estudios en trabajos posteriores para determinar si este rasgo puede ser un índice entonativo determinante en la distinción entre ambas.

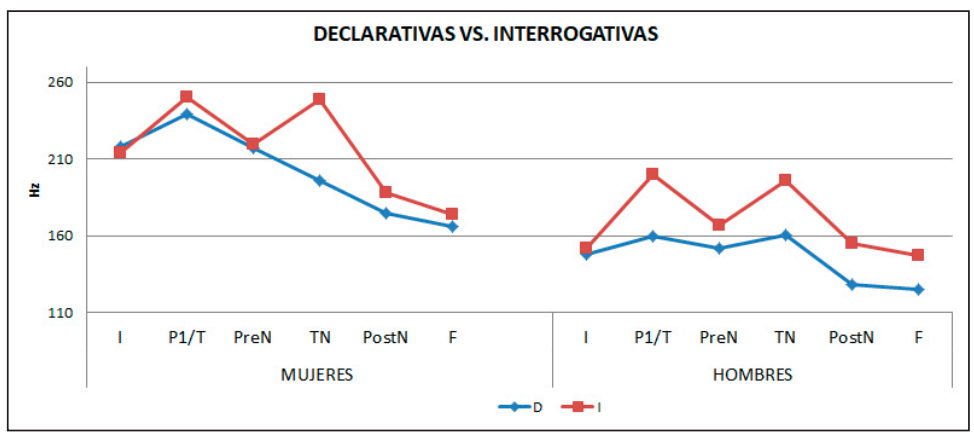

Figura 12. Estilización de los contornos entonativos de las frases declarativas e interrogativas 


\section{CONCLUSIONES}

En este trabajo hemos realizado un estudio fonético-fonológico (según la propuesta de Dorta et al. 2013 a y Dorta y Díaz 2018 a) de oraciones declarativas e interrogativas con acento final llano de un corpus Map task emitido por mujeres y hombres sin estudios superiores procedentes de cuatro regiones venezolanas: Central (Aragua), Los Llanos (Barinas), Los Andes (Mérida) y Sur-Oriental (Bolívar). Los resultados obtenidos nos permiten extraer las siguientes conclusiones más relevantes:

1) Las declarativas venezolanas con final llano presentan diferencias entre los sexos en el núcleo entonativo. Así, en las mujeres hemos registrado la entonación más general en español, esto es, un contorno descendente hasta el final absoluto (/L* $\mathrm{L} \% /)$; en cambio, los hombres usan un patrón circunflejo $\left(/ \mathrm{H}^{*} \mathrm{~L} \% /\right)$, aunque excepcionalmente es descendente o el pico nuclear no tiene un ascenso relevante perceptivamente $\left(/ \mathrm{L}^{*} \mathrm{~L} \% /\right)$. Si bien en las mujeres este comportamiento coincide con el descrito para el corpus experimental (v. gr. Dorta y Díaz 2018 b), no sucede lo mismo en los hombres pues en este último corpus se registraba un pequeño pico nuclear que carecía de relevancia perceptiva al contrario de lo que sucede en el habla más espontánea, donde hemos observado que el pico sí tiene relieve y que el movimiento puede abarcar toda la frase. En definitiva, la mayor espontaneidad del corpus propicia que la configuración del pico adquiera importancia.

2) En las interrogativas, todos los informantes coinciden en utilizar la configuración circunfleja $\left(/ \mathrm{H}^{*} \mathrm{~L} \% /\right)$. No obstante, si el inicio es átono, en las mujeres se da un patrón descendente en un $28,6 \%$ prácticamente centrado en la mujer de Barinas (21,4\%). Los datos del corpus experimental (v. gr. Dorta y Díaz 2018 b) coinciden con los del Map task en todos los informantes, con la excepción de Barinas en que solo se registraba el patrón descendente y no el circunflejo. Por tanto, este último es favorecido en este caso por el habla más espontánea. 


\section{REFERENCIAS BIBLIOGRÁFICAS}

Anderson, Anne H., Miles Bader, Ellen Gurman Bard, Elizabeth Boyle, Gwyneth Doherty, Simon Garrod, Stephen Isard, Jacqueline Kowtko, Jan McAllister, Jim Miller, Catherine Sotillo, Henry S. Thompson y Regina Weinert. 1991. The HCRC Map task Corpus. Language and Speech 78 (4): 351-366.

Beckman, Mary E., Manuel Díaz Campos, Julia Tevis McGory y Terrell A. Morgan. 2002. Intonation across Spanish in the Tones and Break Indices framework. Probus 14: 9-36.

Chela-Flores, Bertha. 1994. Entonación dialectal del enunciado declarativo de una región de Venezuela. Estudios Filológicos 29: 63-72.

Chela-Flores, Bertha y Juan Manuel Sosa. 1999. La representación subyacente de la entonación dialectal del maracucho. Oralia: Análisis Del Discurso Oral 2: 71-86.

Díaz, ChaXiRaXi y JoSEFA Dorta. 2018 a. La entonación de la zona centro-norte de Venezuela a partir del contraste entre distintos corpus. Revista Lingüistica Española Actual (LEA) 40 (2): 163-186.

2018 b: La entonación de Venezuela. En J. Dorta (ed.). La entonación declarativa e interrogativa en cinco zonas fronterizas del español: Canarias, Cuba, Venezuela, Colombia y San Antonio de Texas, pp. 131-158. Frankfurt am Main/Bern/Bruxelles/ New York/Oxford/Warszawa/Wien: Peter Lang Editions. Studien zur Romanischen Sprachwissenschaft und Interkulturellen Kommunikation, Herausgegeben von Gerd Wotjak.

Díaz, ChaXiraXi y Carolina Jorge. 2019. La influencia del corpus de habla en la entonación de la región suroriental venezolana. En J. Dorta (ed.). Investigación geoprosódica. AMPER: análisis y retos, pp. 103-119. Madrid/Frankfurt: Iberoamericana/Vervuert. Col. Lingüística Iberoamericana, 77.

Díaz, Chaxiraxi, Mercedes Muñetón Ayala y Josefa Dorta. 2017. Estudio comparativo de la entonación en habla formal femenina de Caracas (Venezuela) y Bogotá (Colombia). Revista Internacional de Lingüística Iberoamericana (RILI) 29: 237-256.

Dorta, Josefa y ChaXIRAXI DíAZ. 2018 a. El análisis de la F0 y el etiquetaje prosódico en el marco del Sp_ToBI. En J. Dorta (ed.). La entonación declarativa e interrogativa en cinco zonas fronterizas del español: Canarias, Cuba, Venezuela, Colombia y San Antonio de Texas, pp.71-84. Frankfurt am Main/Bern/Bruxelles/New York/Oxford/Warszawa/Wien: Peter Lang Editions. Studien zur Romanischen Sprachwissenschaft und Interkulturellen Kommunikation, Herausgegeben von Gerd Wotjak.

2018 b. Fonética y fonología de los movimientos melódicos en habla rural de Cuba y Venezuela. Revista de Filología Hispánica 34.2: 665-689.

2018 c. Proximidad y distancia prosódica desde el punto de vista acústico entre Canarias y Venezuela. Dialectología 21: 37-60.

Dorta, Josefa, Chaxiraxi Díaz, Beatriz Hernández, Carolina Jorge Trujillo y José ANTONio MARTín Gómez. 2013 a. El marco de la investigación: aspectos metodológicos. En J. Dorta (ed.). Estudio comparativo preliminar de la entonación de Canarias, Cuba y Venezuela, pp. 53-83. Madrid-Santa Cruz de Tenerife: La Página Ediciones S/L, Colección Universidad.

Dorta, Josefa, Chaxiraxi Díaz, Elsa Mora, Carolina Jorge Trujillo y Nelson Rojas. 2013 b. La frecuencia fundamental (F0). En J. Dorta (ed.). Estudio comparativo preliminar 
de la entonación de Canarias, Cuba y Venezuela, pp. 171-216. Madrid-Santa Cruz de Tenerife: La Página ediciones S/L, Colección Universidad.

Estebas Vilaplana, Eva y Pilar Prieto. 2008. La notación prosódica del español: una revisión del Sp_ToBI. Estudios de Fonética Experimental 17: 265-283.

Fernández Planas, Ana María y Eugenio Martínez Celdrán. 2003. El tono fundamental y la duración: dos aspectos de la taxonomía prosódica en dos modalidades de habla (enunciativa e interrogativa) del español. Estudios de Fonética Experimental 12: 165-200.

Hualde, José Ignacio. 2003. El modelo métrico y autosegmental. En Pilar Prieto (ed.). Teorías de la entonación, pp. 155-184. Barcelona: Ariel Lingüística.

López Bobo, M. Jesús, Carmen Muñiz CaChón, Liliana Díaz Gómez, Norberto Corral Blanco, David Brezmes Alonso y Mercedes Alvarellos Pedrero. 2007. Análisis y representación de la entonación. Replanteamiento metodológico en el marco del proyecto AMPER. En J. Dorta (ed.). La prosodia en el ámbito lingüístico románico, pp. 17-34. Madrid/Santa Cruz de Tenerife: La Página ediciones S/L, Colección Universidad.

MÉndeZ, JoRge. 2010. Interacción de los parámetros acústicos duración y frecuencia fundamental en frases declarativas neutras e interrogativas absolutas de Los Andes venezolanos. Estudios de Fonética Experimental 19: 147-164.

Méndez, Jorge, Elsa Mora y Nelson Rojas. 2008. Manifestación acústica de las interrogativas absolutas en los Andes venezolanos. Language Design. Journal of Theoretical and Experimental Linguistics Special Issue 2: 221-229.

MoRA, ELSA. 1993. Entonación interrogativa. Tierra Nueva 6: 75-87.

1996. Caractérisation prosodique de la variation dialectale de l'espagnol parlé au Venezuela. Tesis doctoral, Université d'Aix-en-Provence.

1997. División prosódica dialectal de Venezuela. Omnia 3 (2): 93-99.

Mora, Elsa, Jorge Méndez, Manuel Rodríguez y Nelson Rojas. 2007. AMPERVENEZUELA. En Josefa DORTA (ed.). La prosodia en el ámbito lingüístico románico, pp. 417-428. Madrid/Santa Cruz de Tenerife: La Página ediciones S/L, Colección Universidad.

Obregón, Hugo. 1981. Hacia el estudio de la entonación dialectal del español de Venezuela. Maracay: Universitario Pedagógico Experimental de Maracay.

Pamies Bertrán, Antonio, Ana María Fernández Planas, Eugenio Martínez Celdrán, Alicia Ortega y M. ${ }^{a}$ Cruz Amorós. 2002. Umbrales tonales en español peninsular. En Actas del II Congreso de Fonética Experimental, pp. 272-278. Sevilla: Universidad de Sevilla.

Pierrehumbert, Janet B. 1980. The Phonology and Phonetics of English Intonation. Tesis doctoral. Massachusetts: Massachusetts Institute of Technology.

Prieto, Pilar y Paolo Roseano (eds.). 2010. Transcription of Intonation of the Spanish Language. München: Lincom Europa.

Quilis, ANTONio. 1983. Frecuencia de los esquemas acentuales en español. Estudios ofrecidos a Emilio Alarcos Llorach 5: 113-126.

RieTVELD, Toni y CARLOS GuSSENHOven. 1985. On the relation between pitch excursion size and prominence. Journal of Phonetics 13: 299-308.

Romano, Antonio. 1995. Développement d'un environnement de travail pour l'étude des structures sonores et intonatives de la parole. Mémoire de DEA en Sciences du Langage, Université Stendhal-Grenoble.

Sosa, Juan Manuel. 1991. Fonética y fonología de la entonación del español hispanoamericano. Tesis doctoral, Massachusetts University. 
1999. La entonación del español, su estructura fónica, variabilidad y dialectología. Madrid: Cátedra.

THE HCRC Map task Corpus. 2008. <http://groups.inf.ed.ac.uk/maptask/\#top> [Consulta 02/12/2019].

Villamizar, Thania. 1998. Aspectos prosódicos del habla rural de la Cordillera de Los Andes. Tesis de Maestría en Lingüística, Universidad de Los Andes. 